\title{
Optimization of gallic acid encapsulation in calcium alginate microbeads using Box-Behnken Experimental Design
}

\author{
Kamal Essifi ${ }^{1}$ - Mohammed Lakrat ${ }^{2}$. Doha Berraaouan ${ }^{1}$. \\ Marie-Laure Fauconnier ${ }^{3}$. Ali El Bachiri ${ }^{1}$. Abdesselam Tahani ${ }^{1}$
}

Received: 19 July 2020 / Revised: 23 September 2020 / Accepted: 30 September 2020

(c) Springer-Verlag GmbH Germany, part of Springer Nature 2020

\begin{abstract}
The aim of this study was the optimization of the gallic acid (GA) encapsulation efficiency within calcium alginate microparticles by the ionotropic gelation technique, using Box-Behnken design for the surface methodology response. For this purpose, three independent variables were selected: sodium alginate concentration (X1), calcium chloride concentration (X2), and gallic acid concentrations (X3). The influence of each variable on the encapsulation efficiency was evaluated. The optimum conditions to reach maximum encapsulation efficiency were found to be: $X 1=30 \mathrm{~g} / \mathrm{l}$ $(3 \%, \mathrm{w} / \mathrm{v}), \mathrm{X} 2=21.63 \mathrm{~g} / \mathrm{l}(2.163 \%, \mathrm{w} / \mathrm{v})$ and X3 $=15 \mathrm{~g} / \mathrm{l}(1.5 \%, \mathrm{w} / \mathrm{v})$, respectively. The encapsulation efficiency was determined to be $42.8 \%$. The obtained microbeads were further examined using differential scanning calorimetry (DSC) and Fourier transform infrared (ATR-FTIR), and the inclusion of gallic acid was confirmed. The gallic acid concentration (X3) is the statistically significant factor in the optimization process. In addition, no autoxidation of the gallic acid compound was observed in the formulated calcium alginate microbeads. Scanning electron microscope (SEM) analysis showed that the shape of the particle was spherical for all formulations and their surface is wrinkled. The release study of the gallic acid carried out in an aqueous medium at $\mathrm{pH}$ value 6.8 , showed that the GA release pattern was fast for all systems studied ( $85 \%$ at $20 \mathrm{~min}$ ), and the profile of the release was influenced by the size of the calcium alginate microbeads. The obtained results reveal that the calcium alginate microbeads prepared through the ionotropic gelation technique possess great prominent for gallic acid encapsulation as well as its liberation.
\end{abstract}

Keywords Alginate microbeads · Gallic acid · Box-Behnken design · Ionotropic gelation $\cdot$ Microencapsulation $\cdot$ Release profile

Kamal Essifi

kamal.essifi.lpapc@gmail.com

$\triangle$ Abdesselam Tahani

a1.tahani@ump.ac.ma

Extended author information available on the last page of the article 


\section{Introduction}

Gallic acid (3,4,5-trihydroxybenzoic acid) is a natural phenolic compound, which is widely used for its antimicrobial and antioxidant properties. It is mostly extracted from food such as tea leaves, citrus fruits, mango, bearberry and many others $[1,2]$.

Gallic acid (GA) has numerous biochemical and physiological properties, as antioxidant [2-4], an antimicrobial agent [5], and an antihyperglycaemic agent [6], antiaging, anti-inflammatory activity [7, 8], it also prevents oxidative stress [9], and some kinds of cancer [10]. Nevertheless, GA shows a powerful astringency and high sensitivity to $\mathrm{pH}$, temperature, light, and oxygen which may decrease its possible uses in the functional formulation applications $[11,12]$. In addition, gallic acid, when dissolved in aqueous medium, tends to autoxidize to a dimer or oligomer structure [13, 14].

To overcome its unpleasant flavor and to avoid its autoxidation, gallic acid requires a certain delivery system. More to the point, encapsulation is a promising technique to keep gallic acid's health benefits. Recently, micro and nanoparticles are increasingly applied in pharmaceutical industry as delivery systems. Therefore, encapsulation of GA by zein submicron was previously achieved to develop an active packaging material with a strong antibacterial activity $[13,14]$. Furthermore, inorganic nanosystems including silica nanoparticles and $\mathrm{Fe}_{3} \mathrm{O}_{4}$ were successfully used to transport gallic acid and control its release [15, 16]. To increase its applications in functional formulations, numerous biopolymerbased hydrogel micro and nanobeads were produced to stabilize GA and control its release. Moreover, sodium alginate, $\beta$-cyclodextrin, acetylated starch xanthan, inulin and chitosan were used to encapsulate and deliver gallic acid [12, 17, 18]. Meanwhile, encapsulation of gallic acid through various methods such as spray drying, lyophilization, emulsification, electrospinning and electrospray was studied [12-14, 18-20]. In addition, these previous investigations have shown that the encapsulation efficiency of gallic acid (GA) varies with the technique used.

A high encapsulation efficiency of gallic acid was reported using the spray drying method for native inulin (83\%) and acetylene inulin (75\%). Contrarily, for the native starch and the acetyled starch, these EE values were lower showing $47 \%$ and $57 \%$, respectively. Thus, revealing the effect of the type of wall material on the interaction of GA-polymer [17]. Starch and inulin are both polysaccharides, but they have different structural characteristics. Starch is a glucopyranose with linear and branched areas [21], while inulin is a fructo-oligosaccharide (FOS) that is mainly linear and slightly branched [22]. The encapsulation efficiency of gallic acid in the systems of $\beta$-cyclodextrin, xanthan and chitosane by the lyophilization method and in the silica nanoparticles by the condensation mechanism is $80,77.5,91.07$ and $89.39 \%$ respectively $[16,18]$. These differences in the structure can explain the differences of GA-polymer interactions and hence different encapsulation efficiencies.

Alginate acid as a natural polysaccharide extracted from brown algae possesses many advantages compared to the various polymers adopted for elaborating 
hydrogel micro and nanobeads [12, 23-29]. The alginate macromolecule consists of chains of gluconic acid (G-block) and chains of mannuronic acid (M-block) and chains of mixed sequence of $\mathrm{M}-\mathrm{G}-$ blocks. The sequence and ratio (M/G) of these uronic acids differ depending on the origin of the alginate and can determine the properties of the alginate [29, 30]. It is a natural, biodegradable and biocompatible material which has a large variety of applications in the functional formulations due to its physical-chemistry properties including gelling properties when cross-linked with di or trivalent ions such as calcium and aluminum cation $\left(\mathrm{Ca}^{2+}, \mathrm{Al}^{3+}, \mathrm{Ga}^{3+}, \mathrm{La}^{3+}\right)[31,32]$. Gallic acid encapsulation by sodium alginate was studied by Hyun Jin Park et al. [12], by electrospray technique, who found that the encapsulation efficiency was just over $20 \%$. Although they prevented the loss of gallic acid during the gelling by dissolution in the collection solution the same concentration of gallic acid as that of the sodium alginate solution. Furthermore, they reported that at the gelling process, the encapsulation of the gallic acid by alginate beads reached its maximum levels when sodium alginate polymer is cross-linked in the presence of $\mathrm{Ca}^{2+}$ cation forming the "eggbox" structure. Meanwhile, the free water amount decreased and at the same time, the concentration of GA inside the calcium alginate beads increased. On the other hand, GA amount in calcium alginate beads became higher compared to the collection solution. What is more, GA was released from the beads into the surrounding medium, which led to a reduction in the encapsulation rate [12].

The ionotropic gelation is based on the polyelectrolytes ability to cross-link (in the presence of counter ions to form hydrogel) beads also called as gelispheres. The utilization of expensive and toxic organic solvents in the microencapsulation technique has been drastically reduced due to evolution of ionotropic gelation and hence provides eco-friendly pharmaceutical hydrogel beads [33].

As far as we know, the encapsulation of gallic acid in a promising biodegradable release system for functional applications such as calcium alginate microbeads shows a weak encapsulation efficiency (EE). In addition, the increase of this parameter (EE) in the encapsulation science is necessary to increase the potential uses of the active compounds like gallic acid (GA), and as well as natural polymers such as sodium alginate, hence, an optimization of encapsulation conditions is important.

In the current study, the Box-Behnken design was adopted to evaluate the effects of three selected parameters namely, sodium alginate concentration, calcium chloride concentration, and gallic acid concentration to determine the optimal conditions for the encapsulation of gallic acid (GA) in calcium alginate microbeads using the ionotropic gelation technique. In addition, various analysis techniques such as UV-vis spectrophotometer, ATR-FTIR, differential scanning calorimetry (DSC) as well as scanning electron microscopy (SEM) were used to study and evaluate the GA encapsulation in the calcium alginate microparticles, its chemical stability in the elaborated microparticles and to study the morphology of obtained microbeads. Subsequently, the kinetic release of the gallic acid loaded microparticles at $\mathrm{pH}=6.8$, and the impact of microbeads size on the release profile is also investigated. 


\section{Materials and methods}

\section{Materials}

Sodium alginate (alginic acid sodium salt from brown algae, \#MKBZ4415V, medium viscosity, 5-40 cps of 1\% aqueous solution) was purchased from SigmaAldrich Co. (St. Louis, USA). Gallic acid monohydrate, trisodium citrate and calcium chloride dihydrate were provided by Sigma-Aldrich Co. (St. Louis, USA). Distilled water that was used for the preparation of all samples, has a conductivity less than $2 \mu \mathrm{S}$.

\section{Methods}

\section{Preparing calcium alginate microbeads}

Calcium alginate microbeads containing GA were obtained through the ionotropic gelation technique. Briefly, $1-3 \mathrm{~g}$ of the sodium alginate $(\mathrm{X} 1 \%$, w/v) and $0.5-1.5 \mathrm{~g}$ of gallic acid (X3\%, w/v) were dissolved in $100 \mathrm{ml}$ of water. After full hydration, the mixed solution was left, without stirring, for about $2 \mathrm{~h}$ to remove any air bubbles from the aqueous medium. Gelling solution was prepared by dissolving $\mathrm{CaCl}_{2} \cdot 2 \mathrm{H}_{2} \mathrm{O}$ powder in distilled water $(2-6 \mathrm{~g} / 100 \mathrm{~mL})(\mathrm{X} 2 \%, \mathrm{w} / \mathrm{v})$. A pump was used to transport the mixed solution of the sodium alginate (X1\%, w/v) and gallic acid (X3\%, w/v) through a tube which its end was connected with a polypropylene micropipette tip, the mixture solution $\left(\mathrm{X} 3 \%, \mathrm{X} 1 \%\right.$, w/v) falls into the $\mathrm{CaCl}_{2}$ solution $(\mathrm{X} 2 \%$, w/v) continuously (see Fig. 1).

\section{Box-Behnken optimization design}

The optimization study (EE) was carried out using Box-Behnken design with three levels $(-1,0,+1)$ to evaluate the effect of the three factors (sodium alginate concentration (X1), calcium chloride concentration (X2), gallic acid concentration (X3)) on the encapsulation efficiency (EE) of gallic acid within calcium alginate microparticles. For this purpose, fifteen runs with three replicates were investigated according to this experimental design. The responses measured were encapsulation percentage of the Gallic acid $(Y)$. To evaluate the response factors and their interactions, a second-order polynomial equation was used:

$$
Y=\mathrm{EE}=a_{0}+\sum a_{i} X_{i}+\sum a_{i i} X_{i}^{2}+\sum a_{i j} X_{i} X_{j} \ldots
$$

where EE is the optimized response, $X 1, X 2$ and $X 3$ are the independent parameters, $\boldsymbol{a}_{\mathbf{0}}$ the model intercept, and $\boldsymbol{a}_{\mathrm{i}}$ and $\boldsymbol{a}_{\mathrm{j}}$ are the regression coefficients.

In the present study, we have used the JMP11 software (SAS Institute, USA) to analyze the obtained results and then to select the optimized conditions for an efficiency encapsulation. The input variables of the Box-Behnken design are given in Table 1. 


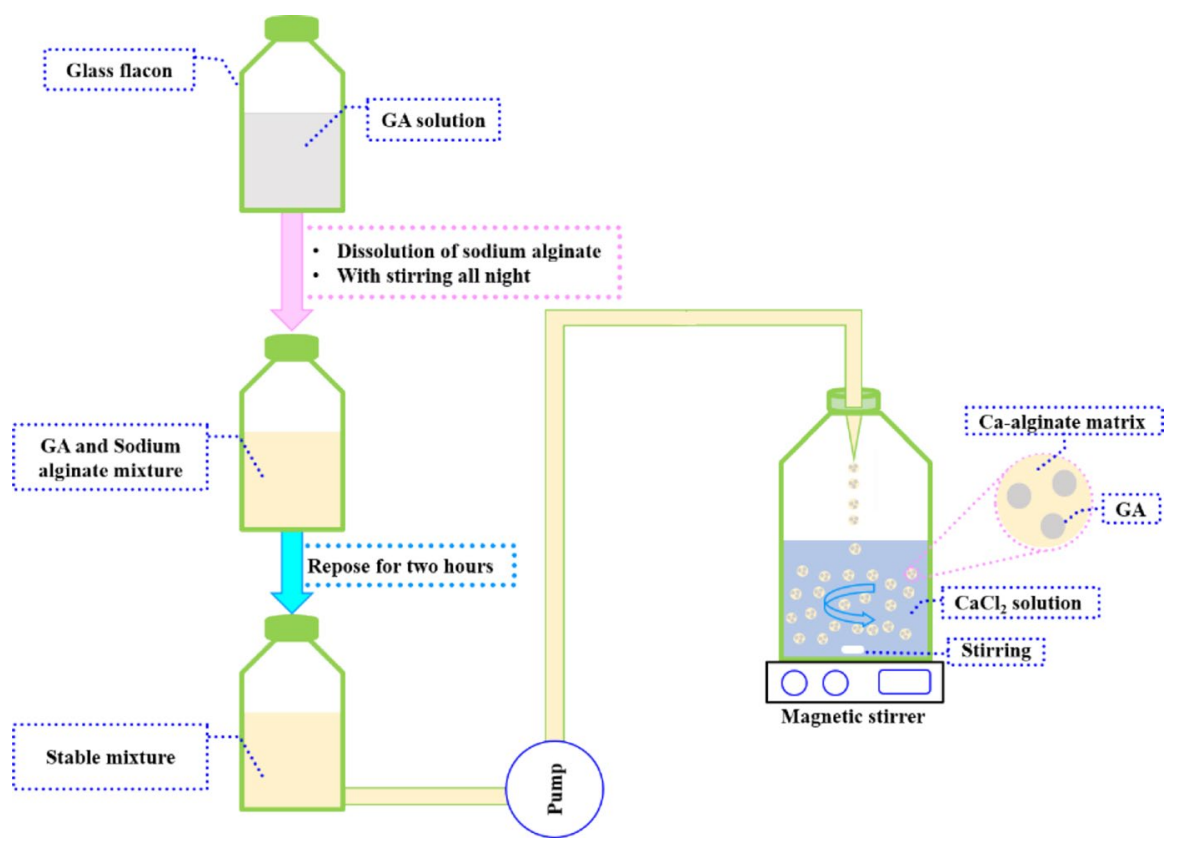

Fig. 1 Scheme of the preparation of gallic acid loaded in calcium alginate microbeads

Table 1 Experimental parameters of Box-Behnken design for preparation of the gallic acid- loaded calcium alginate microbeads

\begin{tabular}{llllr}
\hline Independent variables & Process factors & \multicolumn{2}{l}{ Levels } & \\
\cline { 3 - 5 } & & Low & Middle & High \\
\hline & & -1 & 0 & 1 \\
Sodium alginate concentration $(\% \mathrm{w} / \mathrm{v})$ & $X 1$ & 1 & 2 & 3 \\
Calcium chloride concentration $(\% \mathrm{w} / \mathrm{v})$ & $X 2$ & 2 & 4 & 6 \\
Gallic acid concentration $(\% \mathrm{w} / \mathrm{v})$ & $X 3$ & 0.5 & 1 & 1.5 \\
\hline
\end{tabular}

For any set of variables, the encapsulation efficiency (EE) of GA is calculated using the equation, $\mathrm{EE}=\left(W_{\mathrm{i}}-W_{\mathrm{e}}\right) / W_{\mathrm{i}}$, with $W_{\mathrm{e}}$ is the non-encapsulated amount of gallic acid (GA) (mg), and $W_{\mathrm{i}}$ presents the initial amount of gallic acid (GA) (mg).

The encapsulation amount of GA was evaluated using UV-visible spectrophotometer (UV-1800, China) at $265 \mathrm{~nm}$. For each experiment, $10 \mathrm{mg}$ of microbeads were dissolved in $30 \mathrm{ml}$ sodium citrate solution $(2 \%, \mathrm{w} / \mathrm{v})$, the obtained solution was centrifuged for $5 \mathrm{~min}$ at $8000 \mathrm{rpmn}$. The gallic acid content in supernatant was determined to correct the encapsulation efficiency obtained in the first measure. 


\section{Characterization techniques}

\section{Gallic acid content measurement and loading efficiency}

The amount of GA was determined by the direct measurement of the absorbance using UV-visible spectrophotometer at $265 \mathrm{~nm}$, the gallic acid concentration was obtained through a calibration curve using GA as the standard reference under the same experimental conditions.

Loading capacity was calculated using the following formula:

Loading capacity $=\frac{\text { weight of gallic acid in the calcium alginate microbeads }}{\text { weight of dried calcium alginate microbeads }} \times 100$

The encapsulation efficiency was calculated using the formula below:

$$
\text { Encapsulation efficiency }=\frac{\text { amount of gallic acid in the beads }}{\text { original amount of gallic acid }} \times 100
$$

\section{Attenuated total reflecting-Fourier transform infrared (ATR-FTIR) analysis}

To evaluate the interaction between alginate microbeads and GA as well as to confirm the existence of gallic acid in the microparticles, the ATR-FTIR technique was used. The spectrums were carried out on a Jasco4700-ATR spectrophotometer (Shimadzu, Japan), in the wavelength region between 400 and $4000 \mathrm{~cm}^{-1}$. Furthermore, each spectrum was obtained by averaging 32 scans at a resolution of $4 \mathrm{~cm}^{-1}$.

\section{Differential scanning calorimetry (DSC) analysis}

Thermal properties of sodium alginate polymer, gallic acid, physical mixture of gallic acid and sodium alginate, blank calcium alginate microbeads, and gallic acid loaded calcium alginate microbeads were measured on a model Q100 differential scanning calorimeter (TA Instrument). About 3.0 to $10 \mathrm{mg}$ of sample powder was weighed and sealed in an aluminum DSC pan. After holding isothermally at $0{ }^{\circ} \mathrm{C}$ for $1 \mathrm{~min}$, DSC scanning was carried out from 30 to $350{ }^{\circ} \mathrm{C}$ at a heating rate of $10{ }^{\circ} \mathrm{C} /$ min under dry nitrogen purge of $50 \mathrm{ml} / \mathrm{min}$.

\section{Scanning electron microscopy (SEM) analysis and morphological characterization}

The outer structure of the calcium alginate microparticles obtained under optimal formulation was evaluated by a scanning electron microscope (FEI ESEM "Quanta 200", USA) equipped with an EDX microanalysis. The scanned images were collected digitally, using ETD software. 


\section{GA release study}

The release of gallic acid from the obtained microbeads in water was performed by using UV-visible spectrophotometer. For the analysis, a known amount of microbeads (of about $0.51 \mathrm{~g}$ ) was suspended in $150 \mathrm{~mL}$ of distilled water. The samples were submitted to continuous agitation on an Multi-Position magnetic stirrer (Variomag Poly 15, Germany) operating at $250 \mathrm{rpm}$. At defined time intervals (1-10 min), an aliquot of the supernatant was taken for the measurement of the absorbance at $265 \mathrm{~nm}$. At the end of the measurement, $30 \mathrm{ml}$ of a sodium citrate solution $(2 \%$, $\mathrm{w} / \mathrm{v}$ ) are added and final absorbance is recorded. The value of the final absorbance is corrected by considering the dilution. The experiments were performed in triplicate.

\section{Analysis of gallic acid release kinetics and mechanism}

In order to predict and correlate the gallic acid (GA) release behavior from elaborated calcium alginate microbeads loaded with GA, it is necessary to fit into a suitable mathematical model. The GA release data were evaluated kinetically using various important mathematical models such as zero-order, first-order, and Korsmeyer-Peppas models $[34,35]$.

$$
\text { Zero-order model : } Q=k_{0} t+Q_{0}
$$

where $Q$ represents the active compound released amount in time $t(\min ), Q_{0}$ is the start value of $Q$, and $k_{0}$ is the rate constant.

$$
\text { First-order model }: Q=Q_{0} e^{k_{1} t}
$$

where $Q$ represents the active compound released amount in time $t(\min ), Q_{0}$ is the start value of $Q$, and $k_{1}$ is the rate constant.

$$
\text { Korsmeyer-Peppas model : } Q=k_{p} t^{n}
$$

where $Q$ represents the fraction released at $t(\min ), k_{\mathbf{p}}$ is the rate constant, and $n$ is the diffusional exponent, indicative of gallic acid release mechanism.

Furthermore, the Korsmeyer-Peppas model was employed in the active compound release behavior analysis of these formulations to distinguish between the competing release mechanisms such as Fickian release (diffusion-controlled release), nonFickian release (anomalous transport) and case-II transport (relaxation-controlled release) $[35,36]$.

This model was developed specifically for the release of an active molecule from a polymeric matrix, such as a hydrogel. It is also shown that this equation can adequately describe the release of active compounds or other solutes from slabs, spheres, cylinders, and discs (tablets), regardless of the release mechanism [35]. In addition, to determine the exponent $\mathrm{n}$ and to find out the mechanism of active compound release, it is recommended to use the first $60 \%$ active compound release data. For the release data from several formulations of microcapsules or microspheres, the 
$\mathrm{n}$ value is utilized to characterize different release mechanisms. When $n$ is $\leq 0.43$, it is Fickian release. The $\mathrm{n}$ value between 0.43 and 0.85 is defined as non-Fickian release. When $n \geq 0.85$, it is case-II transport [35-37]. The prediction ability and accuracy of these models were compared by calculation of the squared correlation coefficient $\left(R^{2}\right)$ using OriginPro 2018 software.

\section{Results and discussion}

\section{Effect of the experimental factors on the encapsulation efficiency}

Table 2 shows the mean values for each combination of the three selected parameters and the theoretical predicted values by the mathematical model. Figure 2 reports the effect of each factor on the encapsulation efficiency of gallic acid in calcium alginate microbeads.

Figure 2 indicates that the encapsulation efficiency (EE) of gallic acid is initially inversely proportional to the sodium alginate concentration (X1). For sodium alginate concentrations higher than $2 \%(\mathrm{w} / \mathrm{v})$, an EE increase indicates probably a strong interaction between the phenolic compound and the polymer which promotes the formation of the microbeads with stable structures that consequently have a higher loading capacity. This result about the polymer concentration, agrees with that of REZGUI et al. [38] for valsartan (Angiotensin) encapsulation in poly (lactic acid) (PLA) and poly ( $\varepsilon$-caprolactone) (PCL) polyesters, as well as Behera et al. [39] for encapsulation of fluorouracil (antimetabolite) in ethyl cellulose microspheres who showed that the encapsulation efficiency (EE) increases as well with increasing

Table 2 Experimental conditions of Box-Behnken design and observed response

\begin{tabular}{llllll}
\hline Combination & $X 1(\% \mathrm{w} / \mathrm{v})$ & $X 2(\% \mathrm{w} / \mathrm{v})$ & $X 3(\% \mathrm{w} / \mathrm{v})$ & $\begin{array}{l}\text { Experimental } \\
\text { EE }(\%)\end{array}$ & Predicted EE $(\%)$ \\
\hline$C 1$ & 1 & 4 & 1.5 & 39.44 & 39.04 \\
$C 2$ & 1 & 4 & 0.5 & 18.95 & 18.08 \\
$C 3$ & 1 & 2 & 1 & 25.80 & 27.08 \\
$C 4$ & 1 & 6 & 1 & 26.79 & 26.78 \\
$C 5$ & 2 & 6 & 0.5 & 16.54 & 17.43 \\
$C 6$ & 2 & 6 & 1.5 & 33.44 & 33.85 \\
$C 7$ & 2 & 2 & 1.5 & 39.66 & 38.78 \\
$C 8$ & 2 & 4 & 1 & 27.78 & 27.05 \\
$C 9$ & 2 & 2 & 0.5 & 13.92 & 13.51 \\
$C 10$ & 2 & 4 & 1 & 28.17 & 27.05 \\
$C 11$ & 2 & 4 & 1 & 25.21 & 27.05 \\
$C 12$ & 3 & 4 & 1.5 & 40.75 & 41.62 \\
$C 13$ & 3 & 4 & 0.5 & 20.48 & 20.88 \\
$C 14$ & 3 & 2 & 1 & 29.96 & 29.97 \\
$C 15$ & 3 & 6 & 1 & 30.55 & 29.27 \\
\hline
\end{tabular}




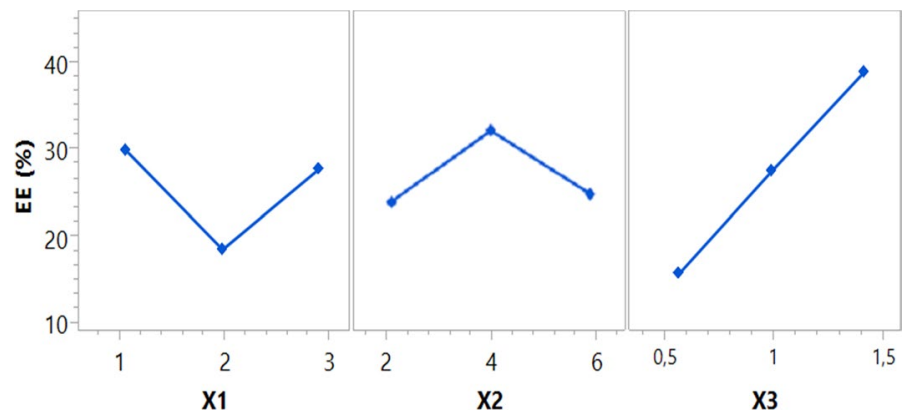

Fig. 2 Influence of sodium alginate concentration (X1\%, w/v), calcium chloride concentration (X2\%, w/v) and gallic acid concentration (X3\%, w/v) on EE mean value

polymer concentration, this polymer concentration effect varies with the variation of structure and nature of polymer [29, 30, 40]. Similar results have been reported in the literature with other drugs [41-43]. For sodium alginate concentration lower than $2 \%$, the observed increase of EE with the decrease of sodium alginate concentration probably indicates the formation of a network structure without homogeneous distribution of pores and their size, which can retard the diffusion of GA towards the external medium (collection solution) during the gelling process.

The calcium chloride concentration (X2) did not show a remarkable effect on EE response, that is, explained by the gelling property of the polymer. It is evident that an increase of calcium chloride concentration can certainly lead to introduce higher levels of $\mathrm{Ca}^{2+}$ ions responsible for cross linking between the alginate chains and consequently forms a dense cohesive structure with a spherical shape and uniform. As a result, can be giving more entrap capacity for the elaborated beads for the molecules in the different size and physico-chemical property compared with the gallic acid molecules [44-46]. However, the calcium chloride concentration did not have a significant effect on the encapsulation efficiency can be attributed to the size and geometrical structure of our encapsulated active compound which is gallic acid.

For the third parameter which is gallic acid concentration (X3), the obtained results show that the encapsulation efficiency (EE) increase with increasing of gallic acid (GA) concentration. This is explained by when we increase the concentration of gallic acid (GA) in the mixture of sodium alginate and GA causes an increase of its concentration in the collection solution $\left(\mathrm{Ca}^{2+}\right.$ solution) when the gelling process. Consequently, prevents the diffusion of the encapsulated GA towards the external medium (collection solution) during the gelling process and thus a better and higher encapsulation efficiency (EE). Over the past 10 years, various types of active compounds have been incorporated successfully in the calcium alginate beads. Most of the active compounds have high water solubility, and therefore it is easier to obtain an homogeneous dispersion of the active compound in alginate solution prior to the encapsulation $[12,47]$. Hence, the probability of diffusion of the mixed active compound with sodium alginate polymer towards the aqueous medium $\left(\mathrm{Ca}^{2+}\right.$ solution) during the encapsulation process was higher, it was mentioned by Nurazreena et al. [47] that generally, an increase in active compound content will 
improve active compound encapsulation efficiency which is confirming our obtained results. Finally, the active compound content is an important factor, which impacts its encapsulation efficiency (EE).

\section{Analysis of experimental data, applied model validation and optimization of the parameters}

\section{Model equation}

To optimize the formulation with the higher EE, the RSM (Response Surface Methodology) was used to predict the correlation between dependent and independent factors. Table 3 shows the coefficient factors of the theoretical model and their related $\mathrm{p}$-values for the analyzed measure. Coefficients with $p$ values less than $5 \%(p<0.05)$ had a noticeable impact on the model prediction efficiency for the obtained response [48]. The polynomial equation representing the relation between the EE response and the independent parameters is described below:

$$
\mathrm{EE}=27.053207+10.424858 X_{3}+2.6160383 X_{1}^{2}-2.211942 X_{2} X_{3}
$$

Coefficients with second-order terms and multiple factors terms characterize the interaction terms and the quadratic relationships in the same order [38]. The coefficients sign shows how the related factors influence the results. If the sign is negative, the response gets lower (antagonist effect), if it is positive the response gets higher (synergetic effect) [49]. Moreover, the results can be repeated without a significant difference as well as the standard error is smaller, and the t-ratio greater than 1.96 (absolute value). In addition, the ratio of estimated values to the standard error represents the t-Ratio.

The Pareto diagram presents the effect of each and their interaction on the encapsulation efficiency (Fig. 3). Furthermore, the effect of each factor on the response is indicated by the length of each bar in the graph.

Table 3 Parameter estimation (note: $*=$ significant value)

\begin{tabular}{lclcc}
\hline Term & Estimated value & Standard error & $t$ Ratio & Prob. $>|t|$ \\
\hline Intercept & 27.053207 & 0.902587 & 29.97 & $<\mathbf{0 . 0 0 0 1 *}$ \\
$(X 1)$ & 1.3452645 & 0.55272 & 2.43 & 0.0591 \\
$(X 2)$ & -0.252034 & 0.55272 & -0.46 & 0.6675 \\
$(X 3)$ & 10.424858 & 0.55272 & 18.86 & $<\mathbf{0 . 0 0 0 1} *$ \\
$(X 1) *(X 2)$ & -0.098978 & 0.781663 & -0.13 & 0.9042 \\
$(X 1) *(X 3)$ & -0.054334 & 0.781663 & -0.07 & 0.9473 \\
$(X 2) *(X 3)$ & -2.211942 & 0.781663 & -2.83 & $\mathbf{0 . 0 3 6 7 *}$ \\
$(X 1) *(X 1)$ & 2.6160383 & 0.813581 & 3.22 & $\mathbf{0 . 0 2 3 6} *$ \\
$(X 2) *(X 2)$ & -1.395315 & 0.813581 & -1.72 & 0.1470 \\
$(X 3) *(X 3)$ & 0.2344484 & 0.813581 & 0.29 & 0.7848 \\
\hline
\end{tabular}


(a)

\begin{tabular}{|c|c|}
\hline & Orthog \\
\hline Term & Estimate \\
\hline$X 3(0.5,1.5)$ & 7.613240 \\
\hline $\mathrm{X} 1 * \mathrm{X} 1$ & 1.346476 \\
\hline $\mathrm{X} 2 * \mathrm{X} 3$ & -1.142242 \\
\hline $\mathrm{Xl}(1,3)$ & 0.982442 \\
\hline $\mathrm{X} 2 * \mathrm{X} 2$ & -0.703302 \\
\hline$X 2(2,6)$ & $-0.184060 \rrbracket$ \\
\hline $\mathrm{X} 3 * \mathrm{X} 3$ & $0.116319]$ \\
\hline $\mathrm{X} 1 * \mathrm{X} 2$ & -0.051112 \\
\hline $\mathrm{X} 1 * \mathrm{X} 3$ & -0.028058 \\
\hline
\end{tabular}

(b)

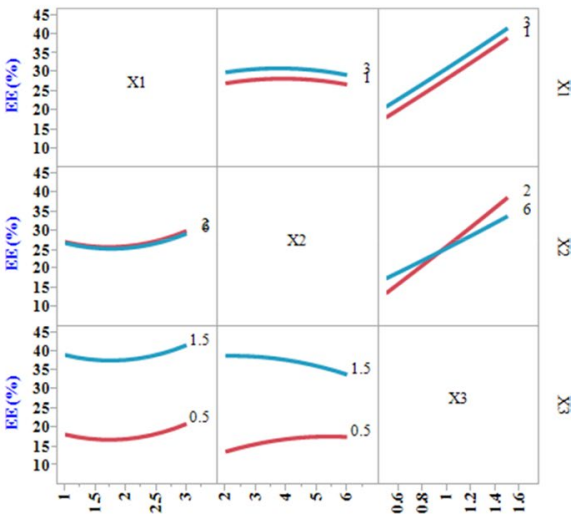

Fig. 3 Pareto plot $\mathbf{a}$ and interaction profiles $\mathbf{b}$, showing the effect of sodium alginate concentration (X1\%, $\mathrm{w} / \mathrm{v})$, calcium chloride concentration $(\mathrm{X} 2 \%, \mathrm{w} / \mathrm{v})$ and gallic acid concentration $(\mathrm{X} 3 \%, \mathrm{w} / \mathrm{v})$ on the (EE) response (Red curve: lower level, Blue curve: higher level of selected factor)

The principal effect of independent variables and their interaction on the encapsulation efficiency is given by the Pareto plot (Fig. 3a). Furthermore, the effect of each factor on the response is indicated by the length of each bar in the graph [38]. In fact, $\mathrm{X} 3$ has the highest influence ( $p$-value $<0.0001)$ and consequently, an antagonistic effect. For $(\mathrm{X} 1)$, the sign is less significant $(p$-value $=0.0591)$, as for the lowest effect was observed for X2 ( $p$ value $=0.6675$ ) (Table 3). In addition, a significant interaction between $\mathrm{X} 1$ and $\mathrm{X} 1(p$ value $=0.0236)$, also between $\mathrm{X} 2$ and $\mathrm{X} 3(p$ value $=0.0367)$ was observed. On the other hand, no significant interaction was detected between $\mathrm{X} 1$ and $\mathrm{X} 2(p$ value $=0.9042), \mathrm{X} 1$ and $\mathrm{X} 3(p$ value $=0.9473), \mathrm{X} 2$ and $\mathrm{X} 2(p$ value $=0.1470)$, and $\mathrm{X} 3$ and $\mathrm{X} 3(p$ value $=0.7848)$ (Fig. 3b).

In the Fig. 4, the 3D plots reveal the effects of each variable and their interactions on the GA encapsulation efficiency. To obtain the observed surfaces, we plotted the obtained EE values against two parameters, holding the third at middle level. Figure 4a shows that at both high and low levels X2 rates, EE increases with X1. Besides, as X2 increases, EE decreases whatever the X1 value.
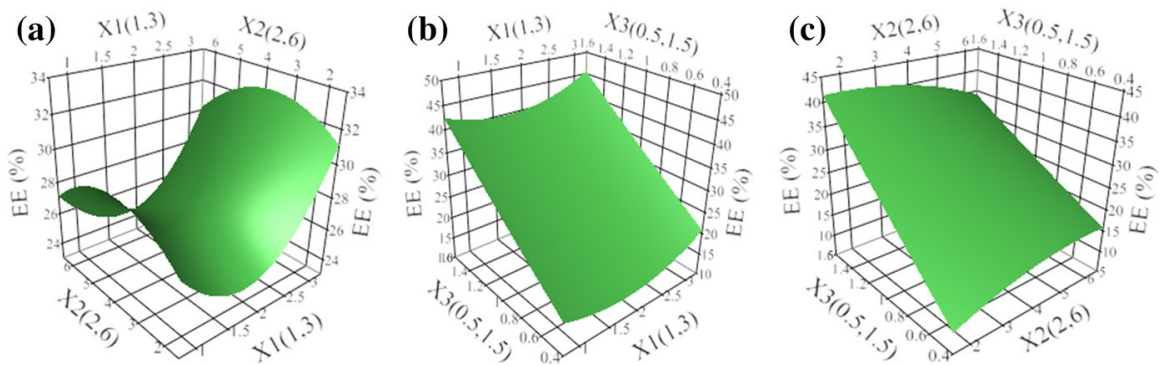

Fig. 4 3D surface plots of EE versus: a X1, X2; b X1, X3 and, $\mathbf{c} X 2, \mathrm{X} 3$ 
Now considering the combined effect of X1 and X3, and as observed in Fig. 4b, EE response increases strongly with X3 whatever the value of X1. Mutually, the same plot displays that EE does not show significant change with $\mathrm{X} 1$ whatever the value of X3. Finally, Fig. 4c shows that EE increases with X3 whatever the value of $\mathrm{X} 2$. Simultaneously, the EE response decreases significantly with X2 if X3 is high, whereas it increases slightly with $\mathrm{X} 2$ if $\mathrm{X} 3$ is low.

In conclusion, among the three independent factors, gallic acid concentration (X3) played the most significant role in the GA encapsulation followed by sodium alginate concentration (X1) and finally calcium chloride concentration (X2) with a negligible role. Which shows that the sodium alginate polymer concentration, as well as the calcium ion concentration has no effect on the porosity of the microbeads for the molecules in the same size and physico-chemical property as gallic acid molecules, and that the increase in the concentration of gallic acid in the mixture causes an increase in its concentration in the collection solution, and consequently prevents the diffusion of the encapsulated GA towards the external medium.

\section{Validation of the model}

The optimization of gallic acid encapsulation efficiency by calcium alginate microparticles was validated by the variance analysis (ANOVA). This step is important to evaluate the meaning and the second-order polynomial equation fit [50]. Several statistical variables given by the software, including lack of fit test, ANOVA and multiple determination coefficients $\left(R^{2}\right)$ tests were used to evaluate the model significance [51]. Table 4 presents the statistical evaluation results obtained.

When the $F$ value is high and the $p$ value is low, the applied model is said to be significant [52]. On the one hand, through $p$ (less than 0.0003) and $F$ values (exceeds 42) the variance analysis shows that the applied model is significant. On the other hand, to reaffirm that the applied model presents a good fitting to the encapsulation efficiency response it is important to report that the lack of fit of the applied model was non-significant $(p>0.05)$ [51]. The checking of the lack of fit applied model was made by the determination coefficient $\left(R^{2}\right)$. Also, the adjusted determination coefficient value was equally high $\left(\operatorname{adj} R^{2}=0.987151\right)$, which more confirms the

Table 4 Variance study (ANOVA) for the applied model

\begin{tabular}{lllccc}
\hline Source & Degree of freedom & Sum of squares & Mean squares & $F$ value & $p$ \\
\hline Model & 9 & 938.84648 & 104.316 & 42.6828 & $<\mathbf{0 . 0 0 0 3}^{*}$ \\
Error & 5 & 12.21995 & 2.444 & & \\
Total & 14 & 951.06644 & & & \\
Lack of fit & 3 & 7.021248 & 2.34042 & 0.9004 & 0.5645 \\
Pure Error & 2 & 5.198707 & 2.59935 & & \\
Model summary statistics & & & & \\
$R^{2}$ & Adj $R^{2}$ & Pred $R^{2}$ & & & \\
0.9945 & 0.987151 & 0.964024 & & & \\
\hline
\end{tabular}


high meaning of the applied model $[53,54]$. The adjusted determination coefficient (adj $\left.R^{2}=0.987151\right)$ for the encapsulation efficiency response is in accord with the predicted determination coefficient value (pred $\left.R^{2}=0.964024\right)$ [55].

Figure 5 presents a comparison between the predicted values obtained by the mathematical model compared with experimental values of encapsulation efficiency response to validate this applied model.

As shown in Fig. 5, a good alignment of the experimental EE (black dots) with the predicted EE (red line) which confirms their correlation. This is confirmed by the determination coefficient $\left(R^{2}=0.9945\right)$, which shows that $99.45 \%$ of the response data is explained by this mathematical model. The correlation coefficient was very close to $1(R=0.995)$ and shows a better correlation between the experimental and the theoretical response predicted by the quadratic model to evaluate the encapsulation process of GA [54].

\section{Optimal factors for the formulation of gallic acid-loaded calcium alginate microparticles}

After studying the effect of independent factors on the encapsulation efficiency, the quadratic regression model was used to determine the optimal values of the selected factors, as well as analyzing the response surface contour plots. Thus, all the factors were chosen in range, and the optimal formulation was the one with the highest gallic acid encapsulation efficiency. The obtained diagram optimization with maximum desirability $(d=0.991082)$, is presented in Fig. 6. The best encapsulation conditions

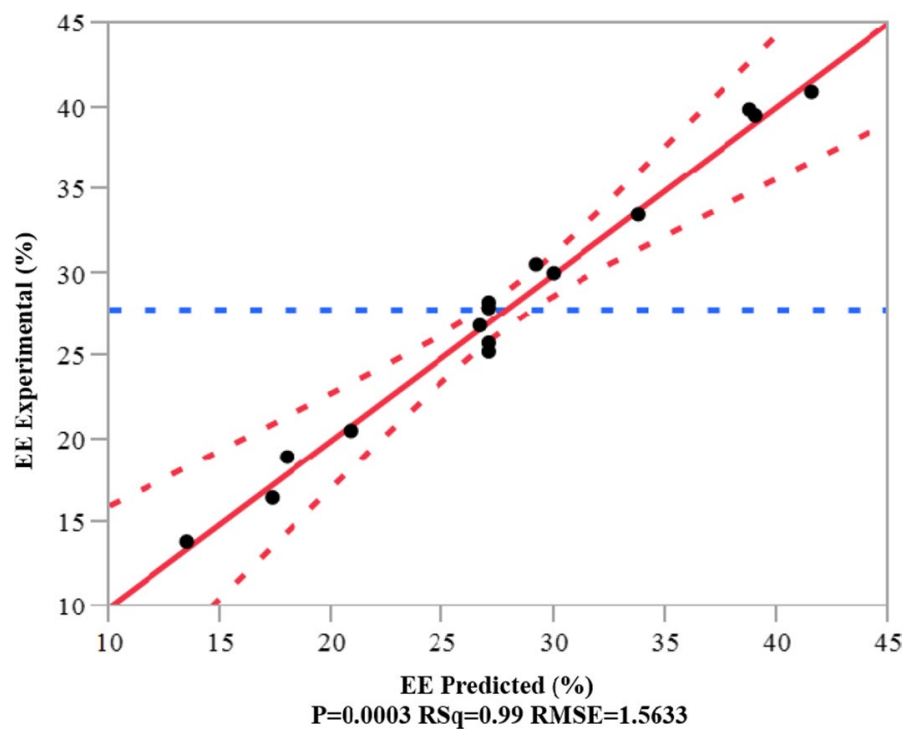

Fig. 5 Plot comparing the experimental and theoretical EE values for gallic acid in calcium alginate microparticles (The red line represents the regression line surrounded by dashed curvilinear boundaries showing the $95 \%$ confidence data distribution limit and the horizontal dashed blue line represents the mean of the experimental EE (\%) value) 


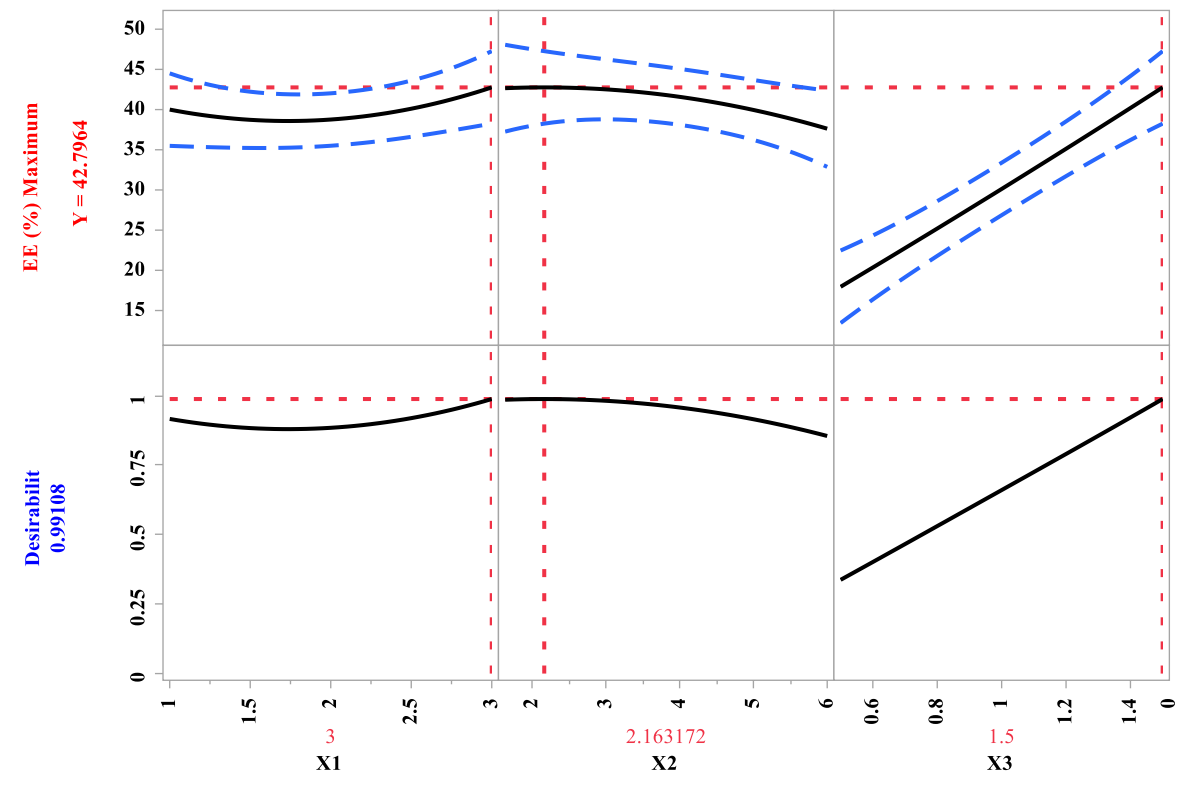

Fig. 6 Schematic optimization of gallic acid encapsulation conditions

obtained for the three selected parameters X1, X2 and X3 are: 3\% (w/v), $2.163 \%$ $(\mathrm{w} / \mathrm{v})$ and $1.5 \%(\mathrm{w} / \mathrm{v})$, respectively. In these optimized conditions, the theoretical encapsulation efficiency of GA obtained by the applied model was found out $42.8 \%$.

In order to validate the quadratic model of the encapsulation study, a checkpoint analysis was carried out in the optimal concentration range. The prepared optimized formulation was indicated by an encapsulation efficiency of $42 \% \pm 0.35$ with a loading capacity of $4 \% \pm 0.035$ (mean $\pm \mathrm{SD}$ ). As can be seen, the EE response obtained was near to the theoretical response $(42.8 \%)$ with a minor error $(1.87 \%)$ proving the accuracy of the obtained theoretical optimum conditions [54]. The obtained lower loading capacity was attributed to the ratio of sodium alginate gallic acid mixture and the weight of gallic acid molecule, Himmelstein et al. [56] showed that the drug loading capacity was directly proportional to the ratio of the amount of drug used during the preparation of microbeads to the total weight of microbeads elaborate. Similar results have been reported by other researchers as well $[12,56]$.

\section{ATR-FTIR analysis}

Figure 7 represents the ATR-FTIR spectra of gallic acid, sodium alginate polymer, mixture of sodium alginate polymer and gallic acid, the blank calcium alginate microbeads and calcium alginate microbeads loaded with gallic acid respectively. Broad vibrational band located at $3273.57 \mathrm{~cm}^{-1}$ was attributed to $\mathrm{O}-\mathrm{H}$ stretching. The two typical band of $\mathrm{COO}^{-}$were observed at $1593.88 \mathrm{~cm}^{-1}$ and $1405.85 \mathrm{~cm}^{-1}$ corresponding to the symmetrical and asymmetrical stretching vibrations, respectively (see spectrums B-D and E in Fig. 7) [12, 23, 57-59]. 


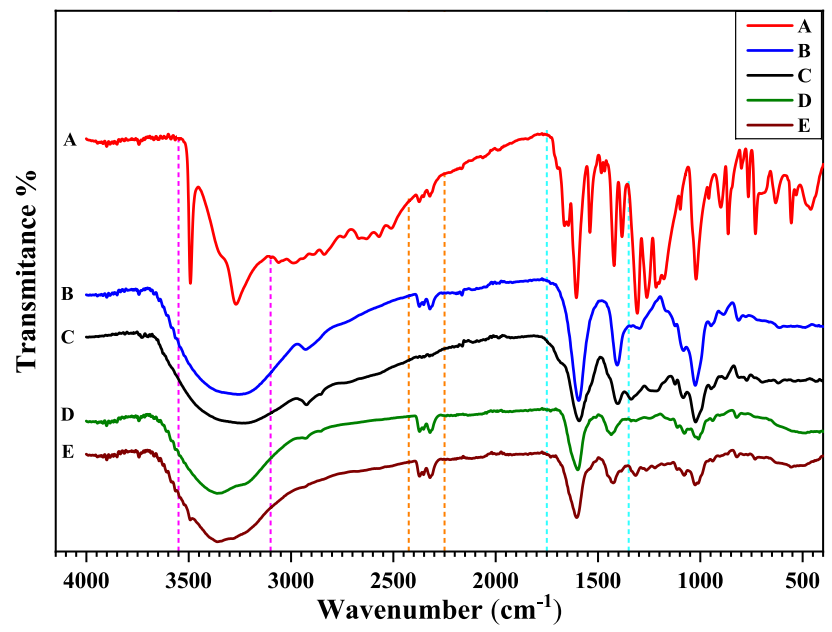

Fig. 7 ATR-FTIR spectra of a gallic acid, b sodium alginate polymer, $\mathbf{c}$ mixture of sodium alginate polymer and gallic acid, $\mathbf{d}$ blank calcium alginate microbeads, e calcium alginate microbeads loaded gallic acid

In the blank calcium alginate microbeads spectrum, a broadband at $3361.32 \mathrm{~cm}^{-1}$ was observed attributed to water molecules $\left(\mathrm{H}_{2} \mathrm{O}\right)$. On the other hand, the two $\mathrm{COO}^{-}$peaks were shifted to higher wavenumber at $1598.70 \mathrm{~cm}^{-1}$ and $1435.74 \mathrm{~cm}^{-1}$ respectively, suggesting that $\mathrm{Ca}^{2+}$ cross-linked alginate at the $\mathrm{COO}^{-}$groups.

The ATR-FTIR spectrum of gallic acid shows small bands between $3060.48 \mathrm{~cm}^{-1}$ and $3491.49 \mathrm{~cm}^{-1}$ corresponding to hydrogen bonds of the $\mathrm{O}-\mathrm{H}$ function. Moreover, bands located at $1605.45 \mathrm{~cm}^{-1}$ and $1307.50 \mathrm{~cm}^{-1}$ represent the $\mathrm{C}=\mathrm{O}$ group. The intense vibrational band at $1021.12 \mathrm{~cm}^{-1}$ was attributed to the vibration of the benzene ring $[12,16]$. Furthermore, the ATR-FTIR spectrum of mixture of sodium alginate polymer and gallic acid present the same bands compared to the calcium alginate microbeads loaded gallic acid with some little differences. In the spectrum of calcium alginate microbeads loaded gallic acid, it is observed that the characteristic bands were shifted to higher wavenumber with no new peak observed, compared to the blank calcium alginate microbeads spectrum, which indicates the incorporation of gallic acid in the microbeads with a possibility of its interaction with calcium alginate microbead, as reported in many previous studies [12, 60]. In addition, as mentioned in several investigations, gallic acid tends more likely to autoxidize, when electrospinning zein fibers loaded with gallic acid, to dimer or oligomer structure $[13,14]$. By using a similar process, our investigation shows that GA is significantly stable in the prepared calcium alginate microbeads.

\section{Differential scanning calorimetry (DSC) analysis}

The thermal behavior of gallic acid, sodium alginate polymer, mixture of sodium alginate polymer and gallic acid, the blank calcium alginate microbeads and calcium alginate microbeads loaded gallic acid were evaluated by differential analysis DSC 


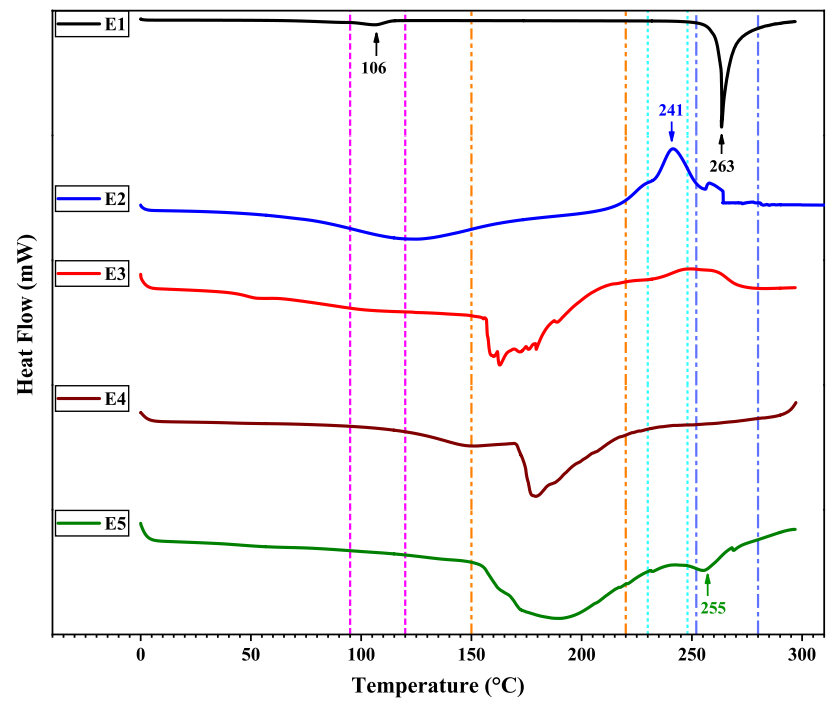

Fig. 8 DSC thermograms of $\mathbf{a}$ gallic acid, $\mathbf{b}$ sodium alginate polymer, $\mathbf{c}$ mixture of sodium alginate polymer and gallic acid, $\mathbf{d}$ blank calcium alginate microbeads, e calcium alginate microbeads loaded gallic acid

and given in Fig. 8. Gallic acid curves show two endothermic peaks, the first one at $106{ }^{\circ} \mathrm{C}$ which is attributed to the loss of free water. The second peak at $263{ }^{\circ} \mathrm{C}$ attributed to the melting of the crystal structure of gallic acid [12, 20]. The significant exothermic peak determined at $241{ }^{\circ} \mathrm{C}$ in the sodium alginate thermogram was attributed to thermal decomposition of sodium alginate polymer (B in Fig. 8) which was absent in the calcium alginate microbeads thermogram (D and E in Fig. 8) [12, 61]. The interaction of gallic acid with the alginate microbeads was confirmed by the endothermic peak at $255{ }^{\circ} \mathrm{C}$ observed in the gallic acid loaded alginate microbeads thermogram. Moreover, no exothermic peak at around $241{ }^{\circ} \mathrm{C}$ as detected in the blank microbeads and gallic acid loaded microbeads thermogram which indicate that the microbeads are more stable compared to sodium alginate as previously reported in [12].

\section{Scanning electron microscopy (SEM) analysis and morphological characterization}

Scanning electron microscopy (SEM) analysis was further employed to examine the morphology of the obtained blank calcium alginate microbeads and calcium alginate microbeads loaded gallic acid under optimal conditions for the studied system and examine the size effect of the microparticles on external surface. Three sizes are studied, $\mathbf{0 . 7} \mathbf{~ m m} \pm \mathbf{0 . 0 3 8}(S 1), \mathbf{0 . 8 5} \mathbf{~ m m} \pm \mathbf{0 . 0 7 2}(S 2)$ and $\mathbf{1} \mathbf{m m} \pm \mathbf{0 . 0 8}(S 3)$ $($ mean \pm SD).

The micrographs observed are shown in Fig. 9. In SEM images, a spherical geometry of microbeads was obtained, regardless of their size. For blank calcium 
(a)

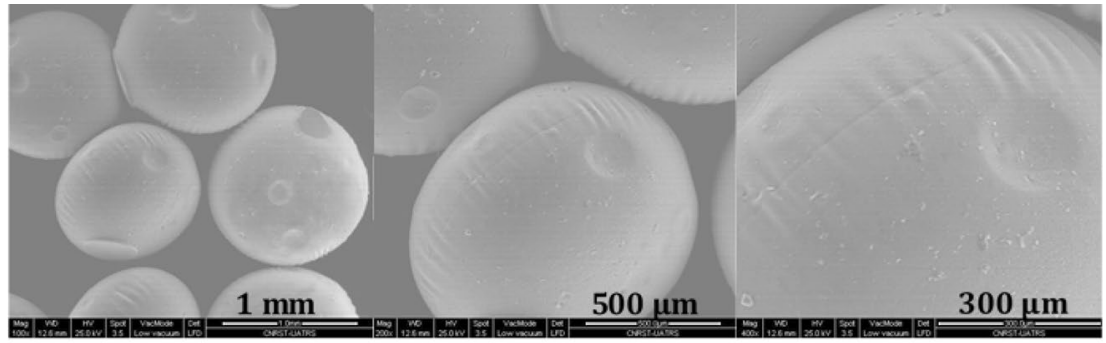

(b)

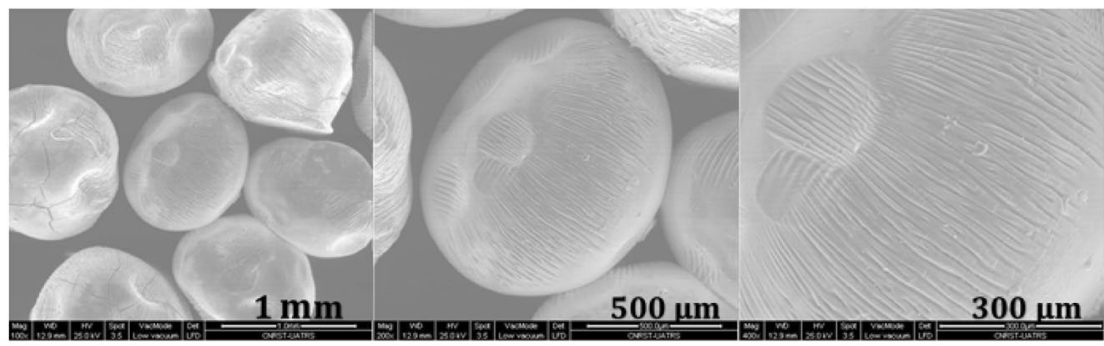

(c)

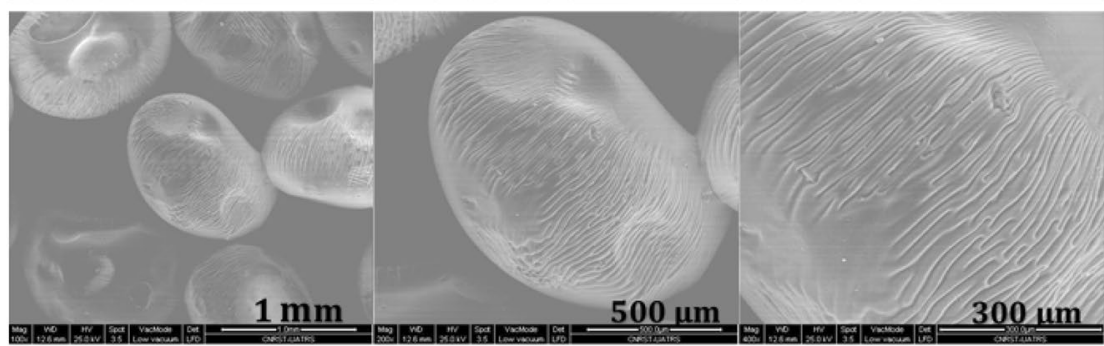

(d)

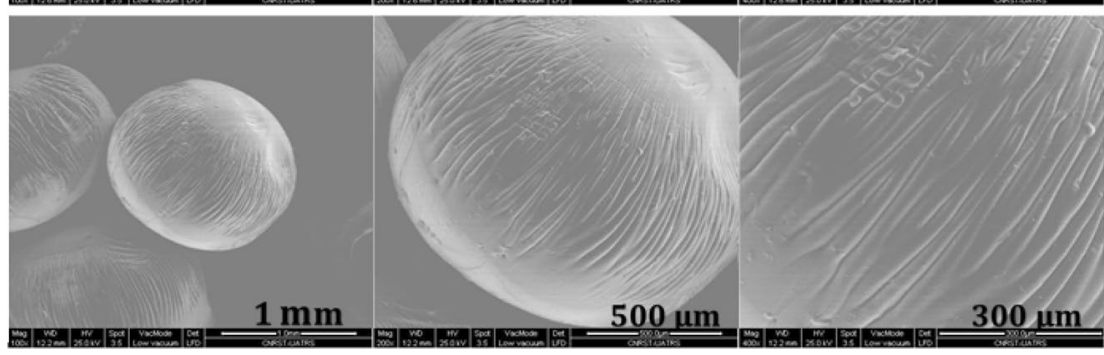

Fig. 9 SEM photographs of calcium alginate microbeads blank a calcium alginate microbeads loaded gallic acid: b S1 $(0.7 \mathrm{~mm})$, c S2 $(0.85 \mathrm{~mm})$ and $\mathbf{d} \mathrm{S} 3(1 \mathrm{~mm})$

alginate microbeads, the microparticles appeared spherical with smooth and homogenized surfaces.

The calcium-alginate microparticles loaded with gallic acid appeared spherical with a wrinkled surface and with uniformly sized microbeads and regular surface texture. In high magnification, a regular alignment of "lines" appears on the surface, which corresponds to the polymer chains with a little difference in the space between the alignment of "lines" that was observed as a function of particle size. 
Fig. 10 Digital photos of (A) wet microbeads of blank calcium alginate, a dried microbeads of blank calcium alginate, (B) wet microbeads of gallic acid loaded calcium alginate (S1), b dried microbeads of gallic acid loaded calcium alginate (S1), (C) wet microbeads of gallic acid loaded calcium alginate (S2), c dried microbeads of gallic acid loaded calcium alginate (S2), (D) wet microbeads of gallic acid loaded calcium alginate (S3), and $\mathbf{d}$ dried microbeads of gallic acid loaded calcium alginate (S3)

The morphological characterization results seen in Fig. 10, show that all obtained calcium alginate microbeads have rigid and spherical and had a smooth surface texture with relatively uniformed distribution sizes. For the wet and dried blank microbeads (without gallic acid) (Fig. 10A and a), the microbeads appeared white with a small size compared to the wet and dried of all obtained microbeads loaded with gallic acid (Fig. 10B-D and b-d), which is explained by the interactions between polymer chains, molecules of water and the GA molecules incorporated in the microbeads matrix.

In addition, the wet gallic acid loaded microbeads appear was milky owing to the GA with milk white color, similar observed results have been reported [12], after dry of all obtained calcium alginate microbeads the shape of the microbeads has similar morphology with that of wet beads, with small size, odorless, and a light yellow color for microbeads loaded with gallic acid as depicted in Fig. 10. This phenomenon can be associated with the release of water molecules (dehydration) and the consequent formation of gallic acid alginate and or gallic acid $\mathrm{Ca}^{2+}$ complexes, which can be yellow colored in the $\mathrm{pH}$ value inside the microbeads.

\section{GA release study}

The release profiles of GA from calcium alginate microbeads ( $S 1, S 2$ and $S 3$ ) obtained under optimal conditions, in distilled water at $25{ }^{\circ} \mathrm{C}$, are presented in Figs. 11 and 12. In the release profile graphs, two steps can be distinguished for all studied systems. The first step showed an initial rapid GA release (85\% within the first $20 \mathrm{~min}$ ), which can be attributed to the presence of discovered gallic acid on the calcium alginate microbeads surface (superficial gallic acid), similar results have been reported by Robert et al. [17]. On the other hand, alginate microbeads encapsulated with a hydrophilic compound or water extract generally have a characteristic of rapid release due to the porous structure of calcium alginate microbeads that did not offer a barrier effect to the encapsulated matter $[62,63]$.

The second step was characterised by a gradual GA release (50-350 min), with shows a lower release rate, this step was attributed to the encapsulated gallic acid released from the calcium alginate microbeads core. In these steps of GA release, the erosion of the polymer matrix due to its dissolution in the release solution was not observed. This could be explained by the release only being studied for a short period, during the long environmental stability of the calcium alginate microbeads.

As it is seen in Fig. 12, the calcium alginate microbeads size showed some control for the GA release, the release speed was rapid in all systems within the first $20 \mathrm{~min}$. The effect of size on the release kinetics of gallic acid was observed, the rate of release decreased when the size of the calcium alginate microbeads was 

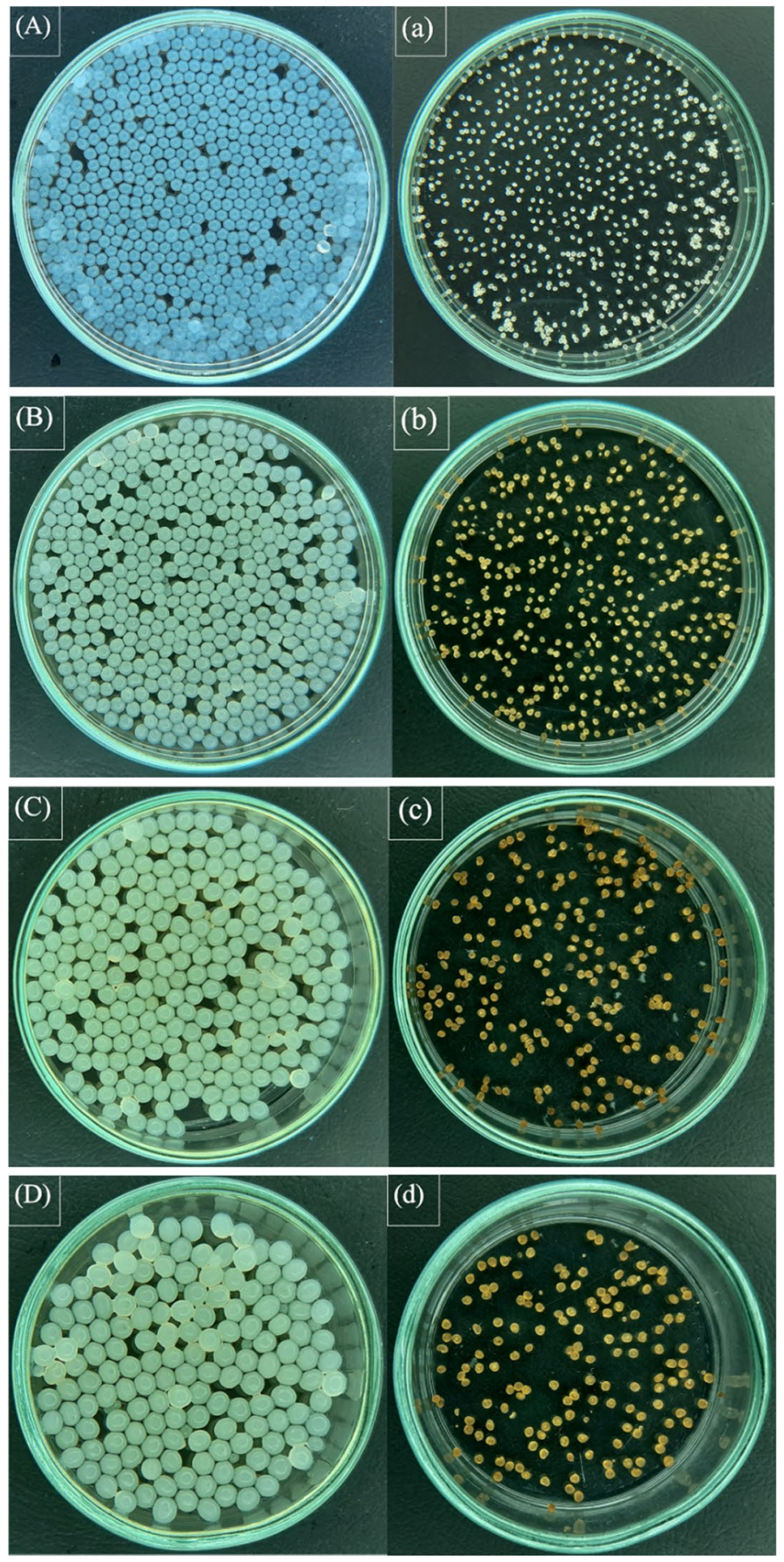


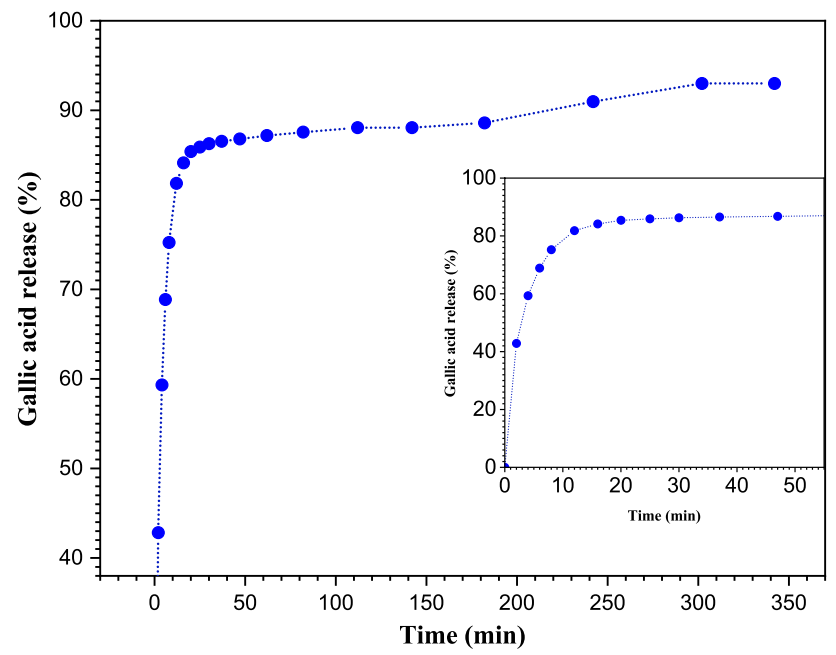

Fig. 11 Release kinetics of gallic acid from alginate microbeads in distilled water at $\mathrm{pH}=6.8$

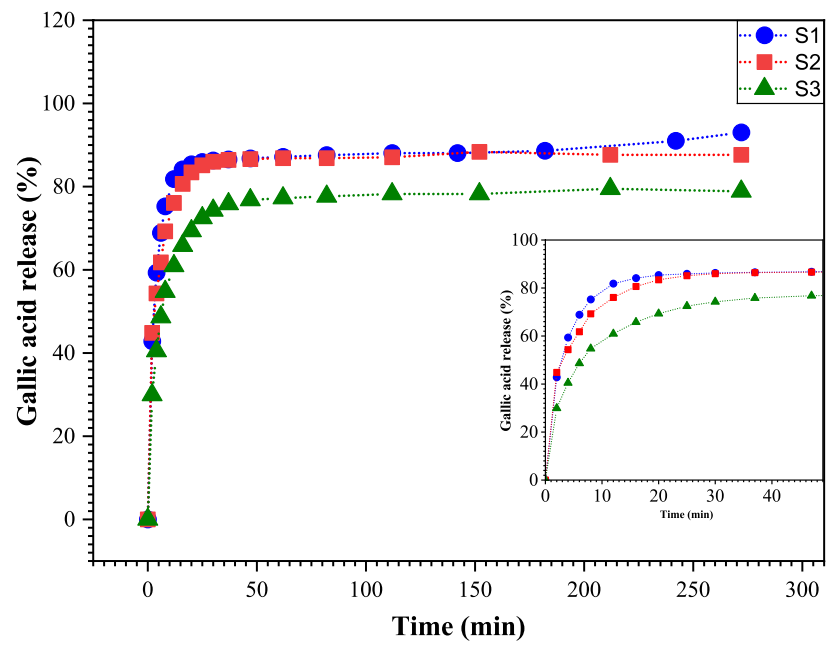

Fig. 12 Effect of the size of the microparticles in the release kinetics of the gallic acid from alginate microbeads in distilled water at $\mathrm{pH}=6.8$

increased, this effect could be observed where the release of the encapsulated acid, which explained by the slow diffusion inside the capsule.

The gallic acid (GA) release from various elaborated calcium alginate microbeads size loaded with GA, it is necessary to fit into suitable mathematical models. The GA release data were evaluated kinetically using various important mathematical models such as zero-order, first-order, and Korsmeyer-Peppas kinetic models. The squared correlation coefficient $\left(R^{2}\right)$ values of these models were calculated for evaluated the prediction ability and accuracy of these models using OriginPro 2018 

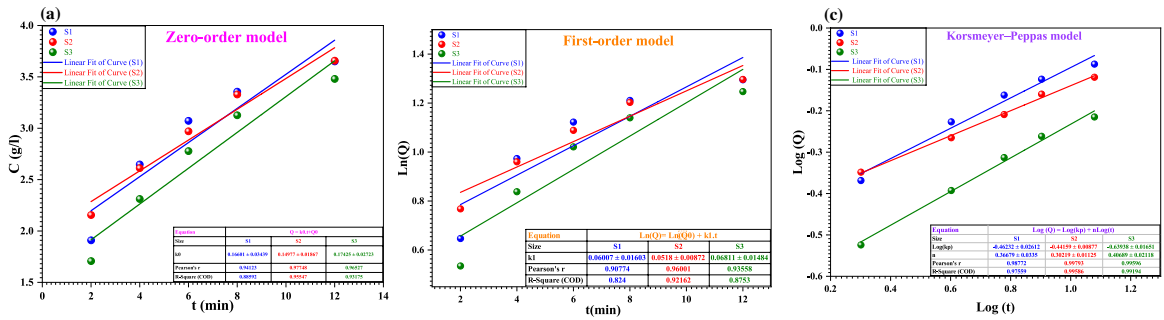

Fig. 13 The fitting of GA release data to zero-order $\mathbf{a}$ first-order $\mathbf{b}$ and Korsmeyer-Peppas $\mathbf{c}$ kinetic models of the three selected sizes (S1, S2 and S3)

Table 5 Release kinetics parameters in distilled water at $\mathrm{pH}-6.8$

\begin{tabular}{|c|c|c|c|c|c|c|c|}
\hline \multirow{3}{*}{$\begin{array}{l}\text { Microbeads } \\
\text { code }\end{array}$} & \multicolumn{7}{|c|}{ Mathematical models } \\
\hline & \multicolumn{2}{|c|}{ Zero-order } & \multicolumn{2}{|c|}{ First-order } & \multicolumn{3}{|c|}{ Korsmeyer-Peppas } \\
\hline & $\overline{R^{2}}$ & $k_{0}$ & $\overline{R^{2}}$ & $k_{1}$ & $\overline{R^{2}}$ & $k_{\mathrm{p}}$ & $n$ \\
\hline$S 1$ & 0.88592 & 0.16601 & 0.824 & 0.06007 & 0.97559 & 0.4349 & 0.36679 \\
\hline$S 2$ & 0.97748 & 0.14977 & 0.92162 & 0.0518 & 0.99586 & 0.3617 & 0.30219 \\
\hline$S 3$ & 0.93175 & 0.17425 & 0.8753 & 0.06811 & 0.99194 & 0.2294 & 0.40689 \\
\hline
\end{tabular}

software. The fitting curves, as well as the obtained release rate constant and correlation coefficient of all formulations fitting by various mathematical models are, respectively, represented in Fig. 13 and Table 5.

The correlation coefficient $\left(R^{2}\right)$ values of GA loaded calcium alginate microbeads neither follows zero order nor first order, but the correlation coefficient $\left(R^{2}\right)$ values were close to the Korsmeyer-Peppas model. Therefore, the release kinetics of gallic acid loaded calcium alginate microbeads follows the Korsmeyer-Peppas model.

The presented data in Table 5 show that the $\mathrm{n}$ values are obtained in the range of 0.30219-0.40689 for all formulation studies indicates the GA diffuses from the calcium alginate microbeads according to Fickian type diffusion process. In the case of the Fickian mechanism, the rate of gallic acid diffusion is much less than that of polymer swelling and relaxation. Similar result was demonstrated by Lopez-Malo et al. [64] when the release of cinnamon essential oil encapsulated in alginate beads and Slaven et al. [65] when the kinetic release of Trichoderma viride spores loaded calcium alginate microspheres. This result helps to understand the release mechanisms of phenolic compounds like gallic acid from calcium alginate microbeads during storage and throughout their use in functional applications.

\section{Conclusion}

In the current work, an optimization study was carried out using experimental design to evaluate the effect of three factors on the encapsulation efficiency of GA. The obtained results show that the gallic acid concentration (X3\%, w/v) has a positive 
effect on the gallic acid encapsulation efficiency and the sodium alginate concentration $(\mathrm{X} 1 \%, \mathrm{w} / \mathrm{v})$ has a smaller significant effect. On the contrary, no significant effect was observed for the calcium chloride concentration $(\mathrm{X} 2 \%$, w/v) on the GA encapsulation efficiency.

ATR-FTIR results show that the gallic acid encapsulated in the calcium alginate microbeads obtained by ionotropic gelation has a good stability, revealing no autoxidation in the spectra of alginate microbeads that is confirmed by DSC. The SEM results carried out on the various size shows that the calcium alginate microbeads appeared spherical with a wrinkled surface and uniform surface texture. In the release study in water (hydrophilic system), the gallic acid released from calcium alginate microbeads has quick release kinetics with a sensitivity to the bead size when releasing the encapsulated gallic acid in the calcium alginate microbeads core. The fast release of the gallic acid from all systems evaluated suggests that the calcium alginate microparticles are preferably used in instant pharmaceutical formulations or in dried products. Fitting of the GA release data to zero-order, first-order, and Korsmeyer-Peppas kinetic models show that the fitting close to the KorsmeyerPeppas model, which is revealed the underlying gallic acid release mechanism as Fickian diffusion kinetics.

Our results suggest that the calcium alginate microparticles formulated by the ionotropic gelation method are a promising system to encapsulate and controlled release water-soluble phenolic organic compounds such as gallic acid to increase further its applications for functional formulations.

Acknowledgements The authors are sincerely thankful to MESRSFC, CNRST-Morocco and UMP for financial support of Project PPR 15-17 and PARA1-2019. The authors are thankful to the Professor Abdelmonaem Talhaoui, Head of Department of Chemistry, University of Mohammed first Oujda, for managing department of analysis.

\section{Compliance with ethical standards}

Conflict of interest The authors report no conflict of interest. The authors alone are responsible for the content and writing of the paper.

\section{References}

1. Soong YY, Barlow PJ (2006) Quantification of gallic acid and ellagic acid from longan (Dimocarpus longan Lour.) seed and mango (Mangifera indica L.) kernel and their effects on antioxidant activity. Food Chem 97:524-530. https://doi.org/10.1016/j.foodchem.2005.05.033

2. Aydogdu A, Sumnu G, Sahin S (2019) Fabrication of gallic acid loaded Hydroxypropyl methylcellulose nanofibers by electrospinning technique as active packaging material. Carbohyd Polym 208:241-250. https://doi.org/10.1016/j.carbpol.2018.12.065

3. Omrani Z, Dadkhah TA (2020) New cyclodextrin-based supramolecular nanocapsule for codelivery of curcumin and gallic acid. Polym Bull 77:2003-2019. https://doi.org/10.1007/s00289-019-02845 $-5$

4. Samooel J, Jun HC, Binna K, Hyejeong Y, Zbigniew AK, Cheorun J (2010) Effect of dietary mixture of gallic acid and linoleic acid on antioxidative potential and quality of breast meat from broilers. Meat Sci 86:520-526. https://doi.org/10.1016/j.meatsci.2010.06.007 
5. Chanwitheesuk A, Teerawutgulrag A, Kilburn JD, Rakariyatham N (2007) Antimicrobial gallic acid from caesalpinia mimosoides lamk. Food Chem 100:1044-1048. https://doi.org/10.1016/j.foodc hem.2005.11.008

6. Punithavathi VR, Prince PSM, Kumar R, Selvakumari J (2011) Antihyperglycaemic, antilipid peroxidative and antioxidant effects of gallic acid on streptozotocin induced diabetic Wistar rats. Eur J Pharmacol 650:465-471. https://doi.org/10.1016/j.ejphar.2010.08.059

7. Arunkumar S, Ilango K, Manikandan RS, Ramalakshmi N (2009) Synthesis and anti-inflammatory activity of some novel pyrazole derivatives of gallic acid. E-J Chem 6:S123-S128. https://doi. org/10.1155/2009/128586

8. Aranya M, Pensak J, Toshihiro A, Worapaka M, Jiradej M (2010) In vitro anti-aging activities of Terminalia chebula gall extract. Pharmaceutical Biology 48:469-481. https://doi.org/10.3109/13880 200903586286

9. Chinmay P, Samik B, Sumanta D, Athar A, Manish G, Iqbal MS, Pallab M, Susanta SA, Bandyopadhyay U (2010) Gallic acid prevents nonsteroidal anti-inflammatory drug-induced gastropathy in rat by blocking oxidative stress and apoptosis. Free Radical Biol Med 49:258-267. https://doi. org/10.1016/j.freeradbiomed.2010.04.013

10. Hsieh-Hsun H, Chi-Sen C, Wei-Chi H, Sheng-You L, Cheng-Hsun W, Chau-Jong W (2010) Antimetastasis effects of gallic acid on gastric cancer cells involves inhibition of NF- $\mathrm{KB}$ activity and downregulation of PI3K/AKT/small GTPase signals. Food Chem Toxicol 48:2508-2516. https:// doi.org/10.1016/j.fct.2010.06.024

11. Fang Z, Bhandari B (2010) Encapsulation of polyphenols-a review. Trends Food Sci Technol 21:510-523. https://doi.org/10.1016/j.tifs.2010.08.003

12. Li J, Kim SY, Chen X, Park HJ (2016) Calcium-alginate beads loaded with gallic acid: preparation and characterization. LWT Food Sci Technol 68:667-673. https://doi.org/10.1016/j.1wt.2016.01.012

13. Yun PN, Simon S, Sudip R, Marija GN, Jianyong J, Conrad OP (2013) Evaluation of gallic acid loaded zein sub-micron electrospun fibre mats as novel active packaging materials. Food Chem 141:3192-3200. https://doi.org/10.1016/j.foodchem.2013.06.018

14. Yun PN, Sudip R, Jianyong J, Marija GN, Michel KN, Dongyan L, Siew YQ (2013) Encapsulation of food grade antioxidant in natural biopolymer by electrospinning technique: a physicochemical study based on zein-gallic acid system. Food Chem 136:1013-1021. https://doi.org/10.1016/j.foodc hem.2012.09.010

15. Dorniani D, Hussein MZB, Aminu UK, Fakurazi S, Abdul HS, Zalinah A (2012) Preparation of Fe3O4 magnetic nanoparticles coated with gallic acid for drug delivery. Int J Nanomed 7:57455756. https://doi.org/10.2147/IJN.S35746

16. Hu H, Nie L, Feng S, Suo J (2013) Preparation, characterization and in vitro release study of gallic acid loaded silica nanoparticles for controlled release. Pharmazie 68:401-405. https://doi. org/10.1691/ph.2013.2205

17. Paz R, Paula G, Reyes N, Chávez J, Santos J (2012) Acetylated starch and inulin as encapsulating agents of gallic acid and their release behaviour in a hydrophilic system. Food Chem 134:1-8. https ://doi.org/10.1016/j.foodchem.2012.02.019

18. Cleonice GdR, Borges CD, Zambiazi RC, Nunes MR, Benvenutti EV, da Luz SR, D’Avila RF, Rutz JK (2013) Microencapsulation of gallic acid in chitosan, $\beta$-cyclodextrin and xanthan. Ind Crops Prod 46:138-146. https://doi.org/10.1016/j.indcrop.2012.12.053

19. Medina-Torres L, Garcĺa-Cruz EE, Calderas F, González Laredo RF, Sánchez-Olivares G, Gallegos-Infante JA, Rocha-Guzmán NE, RodrÍguez-RamÍrez J (2013) Microencapsulation by spray drying of gallic acid with nopal mucilage (Opuntia ficus indica). LWT Food Sci Technol 50:642-650. https://doi.org/10.1016/j.lwt.2012.07.038

20. Nagpal K, Singh SK, Mishra DN (2013) Nanoparticle mediated brain targeted delivery of gallic acid: in vivo behavioral and biochemical studies for protection against scopolamine-induced amnesia. Drug Delivery 20:112-119. https://doi.org/10.3109/10717544.2013.779330

21. Singh N, Chawla D, Singh J (2004) Influence of acetic anhydride on physicochemical, morphological and thermal properties of corn and potato starch. Food Chem 86:601-608. https://doi. org/10.1016/j.foodchem.2003.10.008

22. Stevens CV, Meriggi A, Booten K (2001) Chemical modification of inulin, a valuable renewable resource, and its industrial applications. Biomacromol 2:1-16. https://doi.org/10.1021/bm005642t

23. Peretz S, Anghel DF, Vasilescu E, Florea-Spiroiu M, Stoian C, Zgherea G (2015) Synthesis, characterization and adsorption properties of alginate porous beads. Polym Bull 72:3169-3182. https://doi. org/10.1007/s00289-015-1459-4 
24. Pal P, Edathil AA, Banat F (2019) Calcium alginate gel and hard beads for the removal of total organic acid anions and heavy metal ions from industrial lean methyldiethanolamine solvent. Polym Bull 76:103-118. https://doi.org/10.1007/s00289-018-2376-0

25. Merakchi A, Bettayeb S, Drouiche N, Adour L, Lounici H (2019) Cross-linking and modification of sodium alginate biopolymer for dye removal in aqueous solution. Polym Bull 76:3535-3554. https://doi.org/10.1007/s00289-018-2557-x

26. Li M, Elder T, Buschle-Diller G (2017) Alginate-based polysaccharide beads for cationic contaminant sorption from water. Polym Bull 74:1267-1281. https://doi.org/10.1007/s0028 9-016-1776-2

27. Hu Y, Chen T, Dong X, Mei Z (2015) Preparation and characterization of composite hydrogel beads based on sodium alginate. Polym Bull 72:2857-2869. https://doi.org/10.1007/s0028 9-015-1440-2

28. Guo T, Zhang N, Huang J, Pei Y, Wang F, Tang K (2019) A facile fabrication of core-shell sodium alginate/gelatin beads for drug delivery systems. Polym Bull 76:87-102. https://doi. org/10.1007/s00289-018-2377-z

29. Loh QL, Wong YY, Choong C (2012) Combinatorial effect of different alginate compositions, polycations, and gelling ions on microcapsule properties. Colloid Polym Sci 290:619-629. https ://doi.org/10.1007/s00396-011-2568-8

30. Aarstad O, Strand BL, Klepp-Andersen LM, Skjaǐšk-Bræk G (2013) Analysis of G-block distributions and their impact on gel properties of in vitro epimerized mannuronan. Biomacromol 14:3409-3416. https://doi.org/10.1021/bm400658k

31. Nguyen LQT, Okajima M, Mitsumata T, Kan K, Tran HT, Kaneko T (2012) Trivalent metalmediated gelation of novel supergiant sulfated polysaccharides extracted from Aphanothece stagnina. Colloid Polym Sci 290:163-172. https://doi.org/10.1007/s00396-011-2528-3

32. Berraaouan D, Elmiz M, Salhi S, Tahani A (2017) Effect of calcium chloride on rheological behavior of sodium alginate. Adv Mater Proc 2:629-633. https://doi.org/10.5185/amp.2017/893

33. Rajaonarivony M, Vauthier C, Couarraze G, Puisieux F, Couvreur P (1993) Development of a new drug carrier made from alginate. J Pharm Sci 82:912-917. https://doi.org/10.1002/jps.26008 20909

34. Samaha D, Shehayeb R, Kyriacos S (2009) Modeling and comparison of dissolution profiles of diltiazem modified release formulations. Dissol Technol 16:41-46. https://doi.org/10.14227/ DT160209P41

35. Lamprecht A, Yamamoto H, Takeuchi H, Kawashima Y (2003) Microsphere design for the colonic delivery of 5-fluorouracil. J Control Release 90:313-322. https://doi.org/10.1016/S0168 $-3659(03) 00195-0$

36. de Lima Souza J, Chiaregato CG, Faez R (2018) Green Composite based on PHB and montmorillonite for KNO3 and NPK delivery system. J Polym Environ 26:670-679. https://doi. org/10.1007/s10924-017-0979-4

37. Dash S, Murthy PN, Nath L, Chowdhury P (2010) Kinetic modeling on drug release from controlled drug delivery systems. Acta Poloniae Pharmaceut Drug Res 67:217-223

38. Sadoun O, Rezgui F, G'Sell C (2018) Optimization of valsartan encapsulation in biodegradables polyesters using Box-Behnken design. Mater Sci Eng, C 90:189-197. https://doi.org/10.1016/j. msec.2018.04.041

39. Behera AL, Patil SV, Sahoo SK (2011) Formulation and characteristics of 5 flurouracil microspheres by solvent evaporation method. Int Pharm Pharmaceut Sc 3:32-35

40. Patel YL, Sher P, Pawar AP (2006) The effect of drug concentration and curing time on processing and properties of calcium alginate beads containing metronidazole by response surface methodology. AAPS PharmSciTech. https://doi.org/10.1208/pt070486

41. Maiti S, Dey P, Kaity S, Ray SMS, Biswanath S (2009) Investigation on processing variables for the preparation of fluconazole-loaded ethyl cellulose microspheres by modified multiple emulsion technique. AAPS PharmSciTech 10:703-715. https://doi.org/10.1208/s12249-009-9257-7

42. Venkatesan P, Manavalan R, Valliappan K (2011) Preparation and evaluation of sustained release loxoprofen loaded microspheres. J Basic Clin Pharm 2:159-15962

43. Marquette S, Peerboom C, Yates A, Denis L, Goole J, Amighi K (2014) Encapsulation of immunoglobulin $\mathrm{G}$ by solid-in-oil-in-water: effect of process parameters on microsphere properties. Eur J Pharm Biopharm 86:393-403. https://doi.org/10.1016/j.ejpb.2013.10.013

44. Lotfipour F, Mirzaeei S, Maghsoodi M (2012) Evaluation of the effect of $\mathrm{CaCl}_{2}$ and alginate concentrations and hardening time on the characteristics of Lactobacillus acidophilus loaded 
alginate beads using response surface analysis. Adv Pharmaceut Bull 2:71-78. https://doi. org/10.5681/apb.2012.010

45. Blandino A, Macías M, Cantero D (2001) Immobilization of glucose oxidase within calcium alginate gel capsules. Process Biochem 36:601-606. https://doi.org/10.1016/S0032-9592(00)00240 $-5$

46. Blandino A, Maćas M, Cantero D (2000) Glucose oxidase release from calcium alginate gel capsules. Enzyme Microbial Technol 27:319-324. https://doi.org/10.1016/S0141-0229(00)00204-0

47. Uyen NTT, Hamid ZAA, Tram NXT, Ahmad N (2020) Fabrication of alginate microspheres for drug delivery: a review. Int J Biol Macromol 153:1035-1046. https://doi.org/10.1016/j.ijbio mac.2019.10.233

48. Nagarwal RC, Srinatha A, Pandit JK (2009) In situ forming formulation: development, evaluation, and optimization using 33 factorial design. AAPS PharmSciTech 10:977-984. https://doi. org/10.1208/s12249-009-9285-3

49. Deshmukh RK, Naik JB (2015) The impact of preparation parameters on sustained release aceclofenac microspheres: a design of experiments. Adv Powder Technol 26:244-252. https://doi. org/10.1016/j.apt.2014.10.004

50. Terzioğlu P, Yücel S, Öztürk M (2017) Application of Box-Behnken design for modeling of lead adsorption onto unmodified and $\mathrm{NaCl}$-modified zeolite NaA obtained from biosilica. Water Sci Technol 75:358-365. https://doi.org/10.2166/wst.2016.526

51. Gupta B, Poudel BK, Pathak S, Tak JW, Lee HH, Jeong JH, Choi HG, Yong CS, Kim JO (2016) Effects of formulation variables on the particle size and drug encapsulation of imatinib-loaded solid lipid nanoparticles. AAPS PharmSciTech 17:652-662. https://doi.org/10.1208/s1224 9-015-0384-Z

52. Dahmoune F, Spigno G, Moussi K, Remini H, Cherbal A, Madani K (2014) Pistacia lentiscus leaves as a source of phenolic compounds: Microwave-assisted extraction optimized and compared with ultrasound-assisted and conventional solvent extraction. Ind Crops Prod 61:31-40. https://doi.org/10.1016/j.indcrop.2014.06.035

53. Yetilmezsoy K, Demirel S, Vanderbei RJ (2009) Response surface modeling of $\mathrm{Pb}(\mathrm{II})$ removal from aqueous solution by Pistacia vera L.: box-Behnken experimental design. J Hazard Mater 171:551-562. https://doi.org/10.1016/j.jhazmat.2009.06.035

54. Honary S, Ebrahimi P, Hadianamrei R (2014) Optimization of particle size and encapsulation efficiency of vancomycin nanoparticles by response surface methodology. Pharm Dev Technol 19:987-998. https://doi.org/10.3109/10837450.2013.846375

55. Ali A, Shahriar H, Ahmad RM, Esmaeil D, Houshang AH (2014) Optimization of heavy metal removal from aqueous solutions by maghemite $(\gamma$-Fe2O3) nanoparticles using response surface methodology. J Geochem Explor 147:151-158. https://doi.org/10.1016/j.gexplo.2014.10.005

56. Heller J, Himmelstein KJ (1985) Poly (ortho ester) biodegradable polymer systems. Methods Enzymol 112:422-436

57. Zhao J, Li S, Zhao Y, Peng Z (2019) Effects of cellulose nanocrystal polymorphs and initial state of hydrogels on swelling and drug release behavior of alginate-based hydrogels. Polym Bull. https://doi.org/10.1007/s00289-019-02972-z

58. Salisu A, Sanagi MM, Abu Naim A, Abd Karim KJ, Wan Ibrahim WA, Abdulganiyu U (2016) Alginate graft polyacrylonitrile beads for the removal of lead from aqueous solutions. Polym Bull 73:519-537. https://doi.org/10.1007/s00289-015-1504-3

59. Li J, Lee IW, Shin GH, Chen X, Park HJ (2015) Curcumin-Eudragit ${ }^{\circledR}$ E PO solid dispersion: a simple and potent method to solve the problems of curcumin. Eur J Pharm Biopharm 94:322332. https://doi.org/10.1016/j.ejpb.2015.06.002

60. López Córdoba A, Deladino L, Martino M (2013) Effect of starch filler on calcium-alginate hydrogels loaded with yerba mate antioxidants. Carbohyd Polym 95:315-323. https://doi. org/10.1016/j.carbpol.2013.03.019

61. Soares JP, Santos JE, Chierice GO, Cavalheiro ETG (2004) Thermal behavior of alginic acid and its sodium salt. Eclet Quim 29:57-63. https://doi.org/10.1590/s0100-46702004000200009

62. Ross C, Rangika W, Luz S, MaryAnn A (2006) Synbiotic microcapsules that enhance microbial viability during nonrefrigerated storage and gastrointestinal transit. Appl Environ Microbiol 72:2280-2282. https://doi.org/10.1128/AEM.72.3.2280-2282

63. Lupo B, Maestro A, Gutiérrez JM, González C (2015) Characterization of alginate beads with encapsulated cocoa extract toprepare functional food: Comparison of two gelation mechanisms. Food Hydrocolloids 49:25-34. https://doi.org/10.1016/j.foodhyd.2015.02.023 
64. Paris MJ, Ramírez-Corona N, Palou E, López-Malo A (2020) Modelling release mechanisms of cinnamon (Cinnamomum zeylanicum) essential oil encapsulated in alginate beads during vaporphase application. J Food Eng. https://doi.org/10.1016/j.jfoodeng.2020.110024

65. Jurić S, Đermić E, Topolovec-Pintarić S, Bedek M, Vinceković M (2019) Physicochemical properties and release characteristics of calcium alginate microspheres loaded with Trichoderma viride spores. J Integr Agri 18:2534-2548. https://doi.org/10.1016/S2095-3119(19)62634-1

Publisher's Note Springer Nature remains neutral with regard to jurisdictional claims in published maps and institutional affiliations.

\section{Affiliations}

\section{Kamal Essifi ${ }^{1}$ Mohammed Lakrat ${ }^{2}$. Doha Berraaouan ${ }^{1}$. Marie-Laure Fauconnier ${ }^{3}$. Ali El Bachiri ${ }^{1} \cdot$ Abdesselam Tahani $^{1}$}

1 Physical Chemistry of Natural Substances and Process Team, Laboratory of Applied Chemistry and Environment (LCAE-CPSUNAP), Department of Chemistry, Faculty of Sciences, University Mohamed Premier, Oujda, Morocco

2 Mineral Solid Chemistry Team, Laboratory of Applied Chemistry and Environment (MSC-LCAE), Department of Chemistry, Faculty of Sciences, University Mohamed Premier, Oujda, Morocco

3 Laboratory of Chemistry of Natural Molecules of Gembloux Agro-Bio Tech, University of Liège, Liege, Belgium 